\title{
A low repetition rate, passively modelocked Ti:Sapphire oscillator
}

\author{
Tomasz M. Kardaś, ${ }^{* 1}$ Piotr Wasylczyk, ${ }^{2}$ Wojciech Gadomski ${ }^{1}$ \\ ${ }^{1}$ Department of Chemistry, University of Warsaw, Żwirki i Wigury 101, 02-089 Warszawa, \\ ${ }^{2}$ Institute of Experimental Physics, University of Warsaw, Hoża 69, 00-681 Warszawa
}

Received September 1, 2009; accepted September 28, 2009; published September 30, 2009

\begin{abstract}
We present two configurations of low repetition rate Ti:Sapphire femtosecond oscillators with the Herriott cell. Pulse energies of $50 \mathrm{~nJ}$ at $13.6 \mathrm{MHz}$ and $40 \mathrm{~nJ}$ at the $5.4 \mathrm{MHz}$ repetition rate were achieved. We discuss Herriott cell design as well as the details of laser construction.
\end{abstract}

Mode-locked oscillators are an indispensible source of femtosecond pulses at high repetition [1]. A variety of constructions designed for different applications have emerged during the last two decades. One of the development paths taken recently is towards short cavity, gigahertz oscillators [2], triggered by their suitability for optical metrology, optical frequency combs in particular [3]. The other way leads in the opposite direction, towards low repetition frequencies and thus higher pulse energy [4] - [8].

The nano- and micro-joule femtosecond pulses are of special interest for nonlinear physics, where many optical processes, e.g. in material science and material processing can be studied. At first, laser amplifiers seem to be the choice: high pulse energy, up to several milijoules are available. This, however, comes at a cost of complexity, cost and laborious maintenance. On the other hand, a fraction of a microjoule pulse is enough to significantly improve spectroscopic measurements, high harmonic generation, micromachining, micro manipulation and many more.

The gap between standard (100 MHz) oscillators and laser amplifiers can be filled by extended cavity lasers. Energy as high as $0.5 \mu \mathrm{J}$ from a Ti:Sapphire oscillator at the repetition rate of $6 \mathrm{MHz}$ has already been presented [4]. For the $\mathrm{Yb}: \mathrm{KY}\left(\mathrm{WO}_{4}\right)_{2}$ - based lasers, energy of 1.35 $\mu \mathrm{J}$ at $1 \mathrm{MHz}$ was achieved [5]. The record length of Ti:Sapphire oscillator cavity was about $38 \mathrm{~m}$ resulting in the $4 \mathrm{MHz}$ repetition rate [6]. The stability and behavior of these oscillators is an important issue, awaiting systematic study. In the time domain, a period doubling bifurcation was observed [7] and other effects seem to play an important role in extended cavity laser design.

In this paper we present the construction of an extended cavity oscillator in two configurations: in the first one we achieve pulse energy of $50 \mathrm{~nJ}$ at $13.6 \mathrm{MHz}$, in the other -

*E-mail: kardas@chem.uw.edu.pl
$40 \mathrm{~nJ}$ at the $5.4 \mathrm{MHz}$ repetition rate. We discuss extended cavity design and propose solutions for some of the problems encountered.

The motivation to extend oscillator's cavity is increasing the energy $(\varepsilon)$ of the output pulses. In the simple picture, if the average power $(P)$ is kept constant and repetition rate $\left(f_{\text {rep }}\right)$ decreases, pulse energy scales as

$$
\varepsilon=\frac{P}{f_{\text {rep }}}
$$

Extending the standard Ti:Sapphire oscillator cavity up to around $2.5 \mathrm{~m}$ can be performed by moving away the output coupler. Further cavity lengthening results in a significant drop of the output power, mainly due to diffractional losses [9]. For a several-meter cavity, an element preserving the complex beam parameter $(q)$ has to be used, e.g the Herriott cell [10], [11].

The Herriot cell is a stable two-mirror resonator with beam injection and extraction elements [11]. An injected beam bounces between cell mirrors and its $q$ parameter remains unchanged at the cavity output.

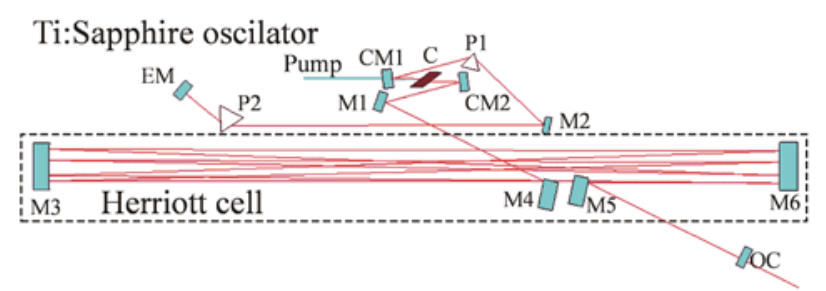

Fig 1. Schematic of the long-cavity femtosecond oscillator. CM1, CM2 - concave mirrors, $\mathrm{f}=50 \mathrm{~mm}, \mathrm{C}-5 \mathrm{~mm}$ Ti:Sapphire crystal, P1, P2 - fused silica prisms, EM, M1, M2 - flat mirrors, OC output coupler (5 or 10\% transmission), M4, M5 - Herriott cell in- and outcoupling mirrors, M3, M6 - Herriott cell mirrors (GVD $\left.=-40 \mathrm{fs}^{2}\right)$.

An extended cavity laser is based on a standard $72 \mathrm{MHz}$ (2.1 m round trip length) oscillator construction [1]. The schematic diagram of our Ti:Sapphire oscillator is shown in Figure 1. The argon-ion pump laser (Coherent Inc. Innova 300C) beam is focused on the $5 \mathrm{~mm}$ Ti:Sapphire crystal with $\mathrm{f}=100 \mathrm{~mm}$ lens. 
There are several parameters to be taken into account while designing the Herriot cavity: mirrors radii, cavity length, number of beam reflections on each mirror, and the number of beam round trips in the cavity. Mechanical constrains must be considered that limit the distance between the mirrors. Using the ABCD matrix formalism one can show that for the Herriott cell with one flat and one concave mirror with radius $R$, the distance between the mirrors $(L)$ has to fulfill

$$
\cos \left(\frac{m \pi}{n}\right)=1-\frac{2 L}{R}
$$

where $m$ is the number of beam reflections from one of the mirrors, and $n / 2$ is the number of beam trips around the cell optical axis. Equation (2) is highly nonlinear and thus increasing the number of reflections will not necessarily increase the total length of the cell, as the corresponding value of $L$ may become small. Moreover, not all combinations of $m$ and $n$ can be realized in practice [10].

We have chosen two configurations: for one flat and one concave mirror with ROC $=2000 \mathrm{~mm}$, the mirrors separation was $1.5 \mathrm{~m}$, there were three reflections $(m=3)$ from each mirror, and the beam traveled around the cell axis once $(n=2)$. For one flat and one concave mirror with $\mathrm{ROC}=3000 \mathrm{~mm}$, the mirrors separation was $1.8 \mathrm{~m}$, the number of reflections was seven and the beam traveled around the optical axis twice $(m=7, n=4)$.

Herriott cell mirrors dispersion was chosen so as to compensate the dispersion of the air. Both mirrors had GVD $=-40 \mathrm{fs}^{2}$, which roughly compensates the GVD of one meter of air under standard conditions around 800 $\mathrm{nm}$. Nevertheless, fine adjustment of the dispersion was necessary in order to obtain mode-locking. This was done using two quartz prisms in the dispersion line.

Injection and extraction into the Herriott cell was realized by two small mirrors (near zero GVD) being two halves cut from a flat half-inch mirror. Since on both Herriott cell mirrors as well as near the center of the cell the laser beams are close to each other, it is convenient to place injecting and extracting mirrors near one fourth of the cell length, closer to the concave mirror. Another commonly used configuration for pulse injection and extraction is through holes in the cell mirrors [7], [8].

Two strategies for aligning the injection and desired pattern of beam spots on the cavity mirrors can be used. One can set an additional output coupler before the injecting mirror, and make adjustments using the short cavity laser beam. Major disadvantage of this approach arises from the fact that the cavity has to be realigned to compensate for additional length after the Herriott cell is added.
Alternatively, one can make use of the laser crystal fluorescence and pump beam spots which are visible even after a few reflections from the IR coated mirrors.

Subsequently, each Herriott cell mirror is adjusted until the desired (circular and symmetric) spot pattern appears on this mirror (rather than on the opposite one). Usually, the alignment of the first few reflections generates the entire pattern automatically.
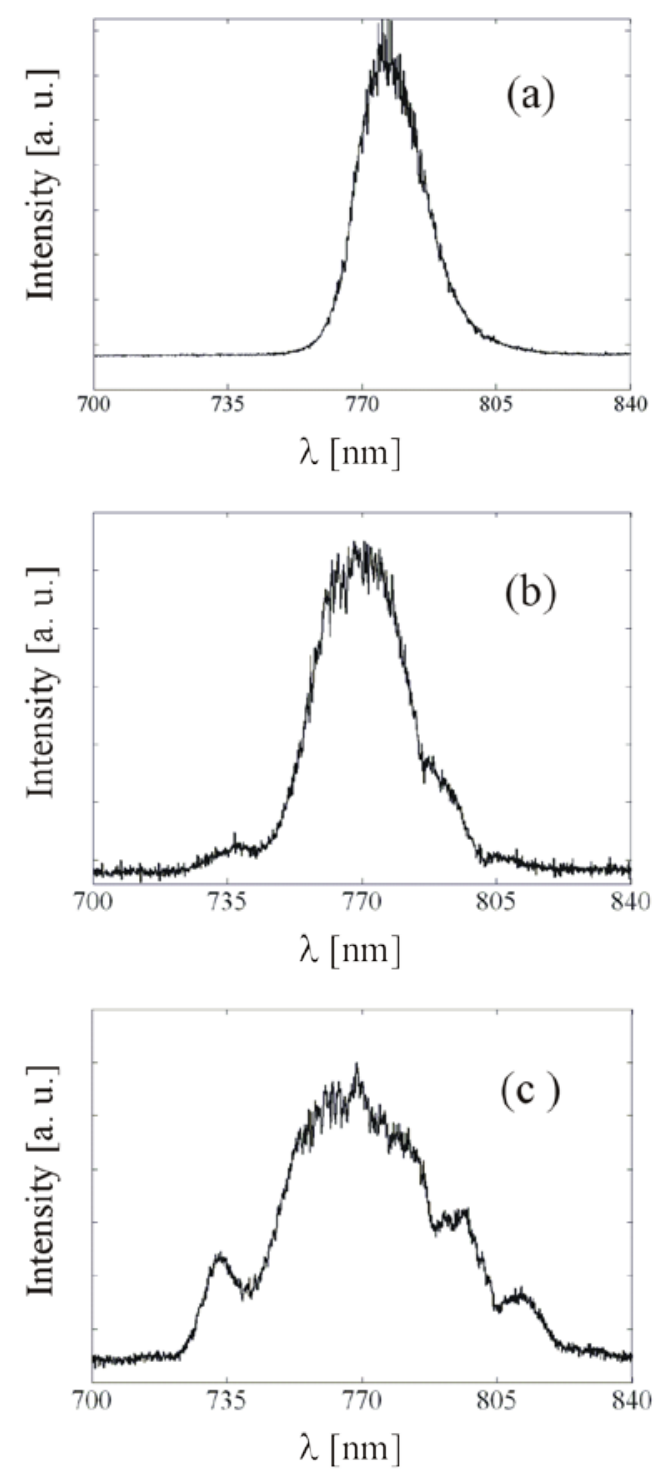

Fig. 2. Measured modelocked laser spectra of the Ti:Sapphire oscillator for three cavity lengths: $\mathrm{a}-2.1 \mathrm{~m}, \mathrm{~b}-11.1 \mathrm{~m}, \mathrm{c}-27.7 \mathrm{~m}$.

It is convenient to find lasing in the extended cavity laser using a low transmission output coupler $\left(\mathrm{T}_{\mathrm{OC}}=5 \%\right.$ in our case), and then switch to higher transmission ( $\mathrm{T}_{\mathrm{OC}}=20 \%$ ) if desired.

The long cavity laser is naturally more sensitive to the air currents but at the same time other parameters of the laser cavity become less critical. For a long cavity 
configuration modelocking was maintained for pump powers between 3.5 and $5.5 \mathrm{~W}$.

We have measured a number of oscillator parameters for two configurations with Herriott cell mirrors of $\mathrm{ROC}=2000 \mathrm{~mm}$ and $\mathrm{ROC}=3000 \mathrm{~mm}$ resulting in 13.6 MHz and $5.4 \mathrm{MHz}$ repetition rate respectively. Table 1. presents the oscillator parameters for configurations with maximal output power achieved for given cavity length. We used output couplers with 5,10 , 12 and $15 \%$ transmission to find that pulse energy only slightly depends on this parameter.

Decreasing the repetition rate from the standard 71.6 $\mathrm{MHz}$ down to $13.6 \mathrm{MHz}$ increases pulse energy by an order of magnitude, even though the pump power had to be reduced to maintain modelocking stability. A further extension of the cavity length to $5.4 \mathrm{MHz}$ does not increase the pulse energy proportionally. We suppose geometrical and diffractive effects may account for this: for $1.8 \mathrm{~m}$ mirror separation the beam becomes large (as it travels $3.6 \mathrm{~m}$ before being reflected from the concave mirror) and the paraxial approximation may not be valid any more (diffraction losses). A large beam can also be partially clipped by cell elements, such as incoupling and outcoupling mirrors (M4 and M5).

Table 1. Oscillator parameters for different cavity configurations: $f_{\text {rep }}$ repetition rate, $T_{O C}$ - output coupler transmission, $L_{\text {cavity }}-$ cavity length, $\mathrm{P}_{\text {Pump }}$ - pump power, $P_{M L}$ - modelocked laser power, $\varepsilon$ - pulse energy, $\delta \lambda_{F W H M}$ - spectral width at half maximum, $\delta \lambda_{5 \%}$ - spectral width at $5 \%$ of the maximum.

\begin{tabular}{|l|l|l|l|}
\hline$f_{\text {rep }}[\mathrm{MHz}]$ & 71.6 & 13.6 & 5.4 \\
\hline$T_{\text {OC }}$ & $5 \%$ & $5 \%$ & $10 \%$ \\
\hline$L_{\text {cavity }}[\mathrm{m}]$ & 2.1 & 11.1 & 27.7 \\
\hline$P_{\text {Pump }}[\mathrm{W}]$ & 6 & 5,5 & 6 \\
\hline$P_{M L}[\mathrm{~mW}]$ & 380 & 680 & 220 \\
\hline$\varepsilon[\mathrm{nJ}]$ & 5.31 & 50.25 & 40.7 \\
\hline$\delta \lambda_{\text {FWHM }}[\mathrm{nm}]$ & 25 & 27 & 40 \\
\hline$\delta \lambda_{5 \%}[\mathrm{~nm}]$ & 55 & 80 & 94 \\
\hline
\end{tabular}

Laser spectra for extended cavity oscillators are usually not as smooth as for standard Ti:Sapphire oscillators [9]. Figure 2. presents normalized spectra measured for three oscillator configurations. Significant spectral broadening is observed for reduced repetition rates and for extended cavity layouts the spectrum can be broadened up to 100 nm FWHM by changing the dispersion line prism insertion. Broad sidebands appear for low repetition rates, however different from the commonly observed Kelly sidebands [12].

A further increase of the oscillator pulse energy can be achieved by setting oscillator parameters to those corresponding to multiple period bifurcations. In the period doubling regime the energy of every other pulse is higher. We have observed a period doubling bifurcation, also reported in [7], in our laser and we aim to maximize pulse energy in this regime.

In conclusion, we have studied two configurations of the modelocked, extended cavity Ti:Sapphire oscillators. By increasing the laser cavity length with the Herriott cell the pulse repetition rate was reduced from $71.6 \mathrm{MHz}$ to 13.6 $\mathrm{MHz}$ and 5.4 MHz. Pulse energies increased by an order of magnitude compared to the standard oscillator and significant spectral broadening was observed.

This work has been supported by the grant No N202.115.31/1463 of the Polish Ministry of Science and Higher Education. P.W. gratefully acknowledges generous support of the Foundation for Polish Science founded by a grant from Iceland, Liechtenstein and Norway through the EEA Financial Mechanism. Project is also operated within the Foundation for Polish Science MPD Programme co-financed by the EU European Regional Development Fund.

\section{References}

[1] D. E. Spence, P. N. Kean, W. Sibbett, Opt. Lett. 16, 42, (1991)

[2] A. Bartels, D. Heinecke, S. A. Diddams, Opt. Lett. 33, 1905, (2008).

[3] T. M. Fortier, A. Bartels, S. A. Diddams, Opt. Lett 31, 1011, (2006).

[4] S. Dewald, T. Lang, C. D. Schröter, R. Moshammer, J. Ullrich, M. Siegel, U. Morgner, Opt. Lett. 31, 2071, (2006).

[5] A. Killi, A. Steinmann, J. Dörring, U. Morgner, M. J. Lederer, D. Kopf, C. Fallnich, Opt. Lett. 30, 1891, (2005).

[6] S. H. Cho, F. X. Kärtner, U. Morgner, E. P. Ippen, J. G. Fujimoto, J. E. Cunningham, W. H. Knox, Opt. Lett. 26, 560, (2001).

[7] A. Fernandez, T. Fuji, A. Poppe, A. Fürbach, F. Krausz, A. Apolonski, Opt. Lett. 29, 1366, (2004).

[8] S. H. Cho, B. E. Bouma, E. P. Ippen, J. G. Fujimoto, Opt. Lett. 24, 1998, (1999).

[9] A. F. González, Chirped Pulse Oscillators: Generating microjoule femtosecond pulses at megahertz repetition rate $(\mathrm{PhD}$ thesis, München 2007).

[10] A. M. Kowalevicz, A. Sennaroglu, A. Tucay Zare, J. G. Fujimoto, J. Opt. Soc. Am. B 23, 760, ( 2006)

[11] A. Sennaroglu, J. G. Fujimoto, Opt. Express 11, 1106, (2003).

[12] S. M. Kelly, Electron. Lett 28, 806, (1992). 